\title{
STS delivery PCL-MECM based hydrogel hybrid scaffold promote meniscal regeneration through controlling the phenotype of macrophages
}

\section{Muzhe Li}

Chinese PLA General Hospital

Han Yin

Chinese PLA General Hospital

Mingxue Chen

Beijing Jishuitan Hospital, Fourth Clinical College of Peking University

Haotian Deng

Chinese PLA General Hospital

Guangzhao Tian

Chinese PLA General Hospital

\section{Zhiguo Yuan}

Shanghai Jiaotong University

\section{Zineng Yan}

Chinese PLA General Hospital

Chao Ning

Chinese PLA General Hospital

Jiang Wu

Chinese PLA General Hospital

\section{Runmin Zhang}

University of South China

Huiyun Li

University of South China

\section{Fu Wei}

University of South China

\section{Wei Chen}

University of South China

\section{Guoliang Yi}

University of South China

\section{Xiang Sui}

Chinese PLA General Hospital

\section{Quanyi Guo}


Chinese PLA General Hospital

\section{Zhiwei Chen}

University of South China

Shuyun Liu ( $\sim$ clear_ann@163.com )

Chinese PLA General Hospital

\section{Research Article}

Keywords: meniscal regeneration, biomaterials, macrophages, M2 polarization, sodium tanshinone IIA sulfonate

Posted Date: February 24th, 2022

DOI: https://doi.org/10.21203/rs.3.rs-1347388/v1

License: (c) (i) This work is licensed under a Creative Commons Attribution 4.0 International License. Read Full License 


\section{Abstract}

\section{Background}

Meniscus injury has a limited ability to heal itself and often results in the progression to osteoarthritis. Tissue engineering offers the possibility of regeneration of severely damaged meniscus. Immunomodulatory strategies based on biomaterials have a wide range of regenerative potential. Macrophages have a high degree of plasticity and will change in morphology and function under the influence of different tissues. M1 macrophages have pro-inflammatory properties, while M2 macrophages have tissue repair and remodeling functions. It has been reported many times that biological scaffolds induce M2 polarization, resulting in the increase of the M2: M1 macrophage phenotypic ratio, thereby promoting tissue repair. However, there is no report on meniscus repair and regenerative.

\section{Results}

In this study, sodium tanshinone IIA sulfonate (STS) delivery Polycaprolactone (PCL)-meniscus extracellular matrix (MECM) based hydrogel hybrid scaffold was fabricated. PCL provides mechanical support, MECM based hydrogel provides a microenvironment conducive to cell proliferation and differentiation, and STS is used to control the phenotype of macrophages. In vitro experiments, we confirmed that STS could not only transform macrophages from M1 to M2 polarization but also prevent meniscal fibrochondrocytes (MFCs) from oxidative stress, apoptosis, and extracellular matrix degradation induced by inflammatory stimulation, thus having stronger proliferation activity. Results of subcutaneous implantation in vivo showed that hybrid scaffold could induce M2 polarization in the early stage. In addition, a hybrid scaffold seeded with MFCs could achieve good meniscus regeneration and chondroprotective effects in the rabbit.

\section{Conclusion}

STS delivery PCL-MECM based hydrogel hybrid scaffold promotes meniscal regeneration through controlling the phenotype of macrophage, which provides a new direction for tissue engineering meniscus regeneration.

\section{Background}

The meniscus is a half-moon tissue on the tibial articular surface that acts as a stress buffer, absorbs shock, and protects joints [1]. The meniscus is not uniform. It may mend after repair because it is surrounded by a blood supply from the joint capsule and synovial artery network in the outside area. Within an ECM that mostly consists of collagen I in the outer area, fibroblast-like cells occur. There is no capacity to heal and regenerate after damage in the inner area owing to a lack of blood transport. Chondrocyte-like cells are the primary cell type in the inner area, encased inside an ECM primarily composed of collagen II (COL-2) and glycosaminoglycans (GAGs). [1]. Meniscus damage has a limited 
capacity to recover and often leads to the advancement of osteoarthritis[2]. To overcome this problem, tissue engineering techniques and other regenerative medicine have been developed. Tissue engineering has been proved to be an advanced therapeutic strategy for meniscus repair[3]. Seed cells, scaffolds, and cytokines are the three essential components of tissue engineering. Previous research has shown that acellular MECM may effectively preserve native meniscus components, perhaps enhancing cell proliferation and accurately regulating endogenous stem cell behavior[4]. Furthermore, owing to its superior permeability to nutrients and metabolites, our team discovered that MECM-based hydrogel, rather than porous MECM scaffold, may produce a microenvironment ideal for cellular adhesion, migration, proliferation, and differentiation[5]. However, hydrogel scaffolds show low biomechanical properties and are difficult to meet the mechanical strength requirements of meniscus scaffolds. PCL scaffolds for bionic meniscus made by 3D printing have good mechanical properties and have been used for meniscus regeneration many times[4-6]. STS, a fat-soluble phenanthrenequinone derivative derived from Salvia miltiorrhiza Bunge (Danshen), has been used to treat coronary heart disease, myocardial infarction, and other conditions [7]. STS has powerful anti-inflammatory, anti-oxidative, and anti-apoptotic effects due to its quinone structure, which is easily redoxed [7]. STS reduces the proliferation and inflammatory cytokine production of synovial fibroblasts from rheumatoid arthritis patients when stimulated by tumor necrosis factor-a(TNF-a)[8]. Importantly, STS prevented articular cartilage degradation by inhibiting apoptosis and inflammatory cytokine expression levels, suggesting that it might be used to treat osteoarthritis (OA)[9-12]. Furthermore, STS has been shown to suppress chondrocyte dedifferentiation $[13,14]$.

The link between biomaterials and the immune system has steadily been uncovered in recent years. Immunomodulatory techniques based on biomaterials offer a high potential for regeneration. These tactics not only reduce unpleasant side effects by delivering medications more efficiently and precisely, but they also actively target and alter resident cell populations[15]. It has been reported numerous times that biological scaffolds induce M2 polarization, resulting in an increase in the M2: M1 macrophage phenotypic ratio, thereby promoting tissue repair in the regenerative neighborhood, implying that M2 polarization plays a significant role in the tissue repair process[16, 17]. There is, however, no information on meniscus repair and regeneration. As a result, the preparation of a biological scaffold with immunomodulatory function, as well as a clear understanding of the immunomodulatory function of the biological scaffold, particularly the critical role of promoting M2 polarization, in meniscus repair and regeneration, may provide new hope for tissue engineering meniscus regeneration. In this study, an STS delivery PCL-MECM based hydrogel hybrid scaffold was fabricated. Mechanical support is provided by $\mathrm{PCL}$, a milieu favorable to cell proliferation and differentiation is provided by MECM-based hydrogel, and STS is employed to alter the phenotype of macrophages. In vitro experiments, we confirmed that STS may not only switch macrophages from M1 to M2 polarization, but also protect MFCs from oxidative damage, apoptosis, and extracellular matrix degradation caused by inflammatory stimulation, resulting in increased proliferative activity. Results of subcutaneous implantation in vivo showed that hybrid scaffold could induce M2 polarization in the early stage. In addition, the hybrid scaffold seeded with MFCs could achieve good meniscus regeneration and chondroprotective effects in the rabbit. (Fig. 1) 


\section{Results}

\section{Effects of STS on cell viability}

The formula for the chemical structure of STS is presented in Figure S2. We evaluated the toxicity of STS to RAW264.7 mouse macrophages and rabbit MFCs using the CCK-8 method. We found that administration of $\operatorname{LG}(5,10,20,40 \mathrm{ug} / \mathrm{ml})$ exhibited no significant effects on RAW264.7 mouse macrophages at $24 \mathrm{~h}$ (Fig. 2A). For rabbit MFCs, STS at the concentrations 5, 10, and $20 \mathrm{ug} / \mathrm{ml}$ exhibited no cytotoxicity at $24 \mathrm{~h}$. However, cell viability was decreased with the treatment of $40 \mathrm{ug} / \mathrm{ml}$ STS (Fig. $2 \mathrm{~A})$. Therefore, STS at the concentrations 5,10 , and $20 \mathrm{ug} / \mathrm{ml}$ was used for subsequent experiments.

\section{STS shifts macrophages from M1 to M2 polarization.}

We performed a series of tests to determine if STS shifts macrophages from M1 to M2 polarization. The cell morphology changes after STS treatment were presented in Figure S3. The result of qRT-PCR showed that RAW264.7 macrophages significantly increased the expression of M1-related genes IL-1 $\beta$ and iNOS after lipopolysaccharide (LPS) stimulation. However, STS decreased the expression of IL-1 $\beta$ and iNOS in a dose-dependent manner (Fig. 2B). Importantly, STS significantly increased the expression of M2-related genes CD206 and Rental (Fig. 2B). Following that, the cytokine secretion properties of polarized macrophages were studied. Two essential upstream inflammatory cytokines, IL-1 $\beta$ and TNF- $\alpha$, are critical effector molecules released by activated M1 macrophages[18]. Results of ELISA showed that STS could significantly inhibit the secretion of IL-1 $\beta$ and TNF-a in M1 macrophages (Fig. 2C). In addition, iNOS (M1 marker) and CD206 (M2 marker) were labeled by immunofluorescence staining. The results showed that STS treatment significantly reduced the fluorescence expression of iNOS in M1 macrophages. Importantly, STS induced high expression of CD206. Compared with the other groups, the LPS + STS10 group had the highest fluorescence expression of CD206, although still lower than that of the M2 positive control (IL-4) (Fig. 2D-E).

\section{STS protects MFCs against the effects of macrophage CM.}

Because STS may greatly decrease the production of pro-inflammatory factors in M1 macrophages, the release of inflammatory factors is reduced, suggesting a hopeful option for further MFC maturation. We treated primary MFCs with macrophage CM. Based on the results of flow cytometry after 3 days of coculture, we found the apoptosis rate was $4.46 \pm 0.44 \%$ in the RAW-CM group, which was greater than the $26.13 \pm 1.95 \%$ in the LPS-CM group. In the LPS + STS5, LPS + STS10, and LPS + STS 20 groups, the apoptosis rate was significantly reduced to $18.4 \pm 1.05 \%, 11.83 \pm 1.88 \%$, and $10.8 \pm 0.36 \%$ respectively, which demonstrated that STS could exert a beneficial effect on macrophage CM-induced MFCs apoptosis, although still lower than that of the M2 positive control (IL-4-CM) (Fig. 3A-B).

\section{STS protects MFCs from IL-1 $\beta$-induced inflammation, oxidative damage, apoptosis, and ECM breakdown.}


TNF- $\alpha$ and IL- $1 \beta$ are two key upstream inflammatory cytokines. TNF- $\alpha$ and IL- $1 \beta$ levels were determined using qRT-PCR and ELISA. We discovered that IL-1 $\beta$ may significantly increase the expression of TNF-a and IL-1 $\beta$ in MFCs. However, this trend can be partly restored by STS (Fig. 4A). Similarly, the results of qRT-PCR and ELISA also showed that STS ameliorated IL-1 $\beta$-induced upregulation of MMP-13, which is a major enzyme targeting the degradation of collagen (Fig. 4A). Furthermore, two apoptosis-related critical genes, Bcl-2 and Caspase-3, were chosen to explore the effects of STS on MFC apoptosis. Bcl-2 inhibits cellular apoptosis, whereas Caspase-3 induces apoptosis[19]. qRT-PCR showed that STS treatment reverses IL-1 $\beta$ inhibited the expression of $\mathrm{Bcl}-2$ and promoted the expression of Caspase-3, thereby protecting MFCs from inflammatory stimulation-induced increased apoptosis (Fig. 4B). Moreover, SOD1, oxidative stress-related genes, also were chosen to evaluate. Western blotting and qRT-PCR demonstrated that STS significantly inhibited IL-1 $\beta$-induced decrease in MRNA and protein expression of SOD1(Fig. 4CD). Collagen II and ACAN are commonly used to evaluate ECM degradation. Immunofluorescence was employed to detect the expression of collagen II and ACAN. As shown in Figs. 4E, STS could reverse the IL-1 $\beta$-induced downregulation of collagen II and ACAN in MFCs.

\section{STS alleviates IL-1 $\beta$-induced inflammation, oxidative stress, apoptosis, and ECM degradation in MFCs partly by inhibiting the IRAK4/TRAF6/NFKB signaling pathway.}

Next, we examined the IRAK4/TRAF6/NFKB signaling pathway to identify the mechanism of the effects of STS on MFCs. First, the results of western blot confirmed that STS significantly inhibited IL-1 $\beta$-induced the expression of p-JNK, p-ERK, and p-p38(Fig.5A-B). In addition, western blot analysis of MFCs also revealed that IL-1 $\beta$ markedly activated the phosphorylation of IKB and p65 in NF-KB signaling. Whereas the phosphorylation of IKB and p65 were decreased by STS, suggesting that the activity of upstream kinases for MAPK pathway and NFKB pathway could be modulated by STS (Fig.5A-B). IRAK4 and TRAF6, known as the upstream enzymes responsible for the MAPK pathway and NFKB pathway, play an important role in the initiation and regulation of inflammatory diseases[20], which are also major mediators in the IL-1 $\beta$-induced inflammatory pathway. As shown in Fig.5A-B, we demonstrated that STS significantly inhibited IL-1 $\beta$-induced upregulation of IRAK4 and TRAF6.

\section{Fabrication and characterization of STS delivery PCL-MECM based hydrogel hybrid scaffold}

Using Solidworks 2018 software, we created a pure PCL scaffold model based on anatomical and imaging data from the rabbit meniscus. 3D printed wedge-shaped porous PCL scaffold with the necessary mechanical characteristics. The model measures $7.73 \mathrm{~mm}$ in length, $5.66 \mathrm{~mm}$ in width, and 1.3 $\mathrm{mm}$ in height. The scaffold's fiber orientation is based on the arrangement of natural collagen fibers in the meniscus (Fig. 6A). Detailed parameters of the printing process are provided in Table S1. The characterization results for Inner MECM and outer MECM are shown in Figure S4. Previous studies in our laboratory confirmed that $2 \%$ MECM based hydrogel was more conducive to maintaining the activity of MFCs and promoting cell proliferation. In addition, $2 \%$ MECM based hydrogel can better promote the secretion of collagen and GAG in MFCs, which means that $2 \%$ MECM based hydrogel can well simulate the microenvironment of MFCs and promote the high expression of specific genes [5]. Therefore, $2 \%$ of MECM was chosen to prepare STS delivery MECM based hydrogel hybrid solutions. In vitro experiments, 
we found that STS10 had the highest polarization capacity of M2(Fig. 2). Therefore, TST10 was used to make STS releasing hybrid scaffold. Inner MECM based hydrogel hybrid solutions and outer MECM based hydrogel hybrid solutions of sustained-release STS were injected into the inner and outer sides of the PCL scaffold respectively. The hybrid scaffold was then fabricated crosslinked with $\mathrm{CaCl}$ (Fig. 6C). To assess the STS releasing behavior, the release rate of STS from the hybrid scaffold was detected in vitro. It was revealed that STS had a relatively high rate of drug release in PBS on the first day, with a cumulative drug release rate of about $56 \%$. The release subsequently slowed (Fig. 6D). Finally, we evaluated the biocompatibility of the scaffold. It was observed from the 3D reconstructed image that the cells were evenly distributed in the MECM-based hydrogel, indicating that the cells were well encapsulated in the hydrogel. Most cells appear fluorescent green (living) with a small number of red fluorescent spots (dead) (Fig. 6E).

\section{In vivo assessment of the early host response and macrophage polarization of mixed scaffolds.}

A number of tests were carried out to investigate the early host response and macrophage polarization of mixed scaffolds. All specimens were coated with fibrocystic after 7 days of implantation, although the fibrocystic in the PCL-hydrogel-STS10 group was whiter and thinner than that in the PCL-hydrogel group. $\mathrm{H} \& \mathrm{E}$ results revealed that both groups had evident infiltration of inflammatory cells at 7 days. (Fig. 7A). Immunohistochemical staining for the pro-inflammatory cytokines IL-1 $\beta$ can reflect the level of inflammation. We found that, compared with the PCL-hydrogel group, the expression level of IL-1 $\beta$ was significantly reduced in the PCL-hydrogel-STS10 group, indicating a lower level of inflammation (Fig. 7A). We utilized immunofluorescence labeling for CD206, a particular marker of M2 macrophages, to further investigate the mechanism through which STS regulates inflammation in vivo. We found that there were more M2 macrophages in the PCL-hydrogel-STS10 group compared with the PCL-hydrogel group at 7 days. (Fig. 7B) The above results confirm that STS delivery PCL-MECM based hydrogel hybrid scaffold promotes the M2 polarization in vivo at an early stage.

\section{In vivo repair}

To fully evaluate the ability of the hybrid scaffold to promote meniscal healing, we implanted them in a critical-size rabbit meniscal defect model established by medial meniscectomy (Figure S5). The negative control group (negative control), PCL-MECM based hydrogel hybrid scaffold group (PCL-hydrogel-MFCs), STS delivery hybrid scaffold group (PCL-hydrogel-MFCs-STS10), and the positive control group (positive control) were chosen for in vivo evaluation. At 3 months after implantation, all scaffold groups regenerated neo-menisci, whereas the positive control group had no neo-meniscus (Figure S6). Further study, such as histological staining, biochemical and biomechanical assessment, could not be done because there was no neo-meniscus in the positive control group. H\&E staining of the neo-meniscus showed that, compared with the PCL-hydrogel-MFCs group, in the PCL-hydrogel-MFCS-STS10 group, more abundant long-forming fibroblast-like cells and round chondrocyte-like cells were observed in the outer and inner regions, respectively. Furthermore, TB staining in the inner region was strongly positive in the PCL-hydrogel-MFCs-STS10 group. More abundant round chondrocyte-like cells have also been observed in the PCL-hydrogel-MFCs-STS10 group compared to the PCL-hydrogel-MFCs group (Fig. 8A). 
Collectively, this comparison indicated the formation of heterogeneous neo-meniscus tissues in the PCLhydrogel-MFCs-STS10 group at 3 months, which is the most similar to the native meniscus. The histological scores determined according to the Ishida scoring system were significantly higher in the PCL-hydrogel-MFCs-STS10 group than in the PCL-hydrogel-MFCs group at 3 months (Fig. 8C). However, the scores were still lower in the PCL-hydrogel-MFCs-STS10 group than in the negative control group, which indicates that there were still some gaps between the repaired tissue in the PCL-hydrogel-MFCsSTS10 group and the native meniscus.

H\&E staining and mankind's ratings were used to assess the chondroprotective effect of the regenerated meniscus. Results of H\&E staining revealed that the articular cartilage surface was better preserved in the PCL-hydrogel-MFCs-STS10 group than that in the PCL-hydrogel-MFCs group and positive control over 3 months, which similar to the macroscopic assessment (Fig. 8B). Meanwhile, mankind's scores in the PCLhydrogel-MFCs-STS10 group were significantly better than the PCL-hydrogel-MFCs group and positive control, although still lower than that of the negative control group (Fig. 8E).

The compressive modulus was also measured at 3 months to assess the mechanical properties of the restored tissue. Results show that compressive modulus of the repaired tissue was greater in the PCLhydrogel-MFCs-STS10 group than in the PCL-hydrogel-MFCs group, although there was no significant statistical difference (Fig. 8D).

\section{Discussion}

The interaction between biomaterials and the immune system is considered to be an important factor affecting material-mediated tissue regeneration. Appropriate manipulation and control of the immune response to make the best use of the inherent healing process is key to tissue repair. Several studies have shown that by modulating immune responses, the results of created tissues may be considerably improved[21-23]. However, as far as we know, this is the first time that immune modulation has been used to improve tissue engineering outcomes in the meniscus regeneration area. Macrophages have a high degree of plasticity and will alter in shape and function as a result of interactions with other tissues. Macrophages are classified into two types: M1 and M2. M1 macrophages are pro-inflammatory, whereas M2 macrophages are involved in tissue repair and remodeling[24]. Following biomaterial implantation, a local nonspecific immune response caused scaffold breakdown, an early event required for the repair process, involving a continual influx of neutrophils and classically activated M1 macrophages. Following that, the infiltrated M1 macrophages transform into M2 macrophages, which express CD163, CD206, and key wound-healing genes like arginase and retnal, as well as secrete IL-10, TGF-b, IL-1RA, chemokine (CeC motif) ligand 18 (CCL18), and other anti-inflammatory mediators, eventually leading to tissue regeneration[17]. M2 macrophages have been proved to not only promote BMSCs invasion, migration, proliferation, and chondrogenesis of bone marrow mesenchymal stem cells but also increase the expression of COL II in chondrocytes, thus promoting the maturation of tissue-engineered cartilage [22, 25]. Furthermore, phenotypic shifts from $M 1$ to $M 2$ are not always apparent. It is determined by the source of the tissue, the technique of sterilization, and the chemical cross-linking agent [25]. The presence 
of M1 macrophages for an extended period of time results in the generation of cytotoxic reactive oxygen species [26]. Furthermore, extending inflammatory fibrous sacs reduces the biomaterial's capacity to induce tissue development or breakdown as intended[27]. To enhance tissue healing, a successful shift to M2 macrophages is required. We focused on the effects of STS on macrophage repolarization in our work and discovered that STS therapy might repolarize M1 macrophages to the M2 phenotype (Fig. 2BD). Furthermore, we were ecstatic to learn that STS might reduce macrophage CM-induced MFC apoptosis, albeit to a lesser extent than the M2 positive control (IL-4-CM) (Fig. 3A-B). The early macrophage phenotype following material implantation is the deciding element in biological scaffold remodeling[28]. M1 macrophages developed first after transplantation, followed by M2 macrophages. According to research, macrophages can switch from M1 to M2 polarization 11 days after transplantation. Further research revealed that partial inhibition of macrophages at the early stage of transplantation, namely M1 macrophages, encouraged greater cartilage matrix accumulation[22]. As a result, we examined the early host response and macrophage polarization of hybrid scaffolds after 7 days of subcutaneous implantation in our study. In the early stages of the scaffold, we discovered that STS Delivery PCL-MECM based hydrogel hybrid scaffold increased M2 polarization and blocked IL-1 $\beta$ production (Fig. 7).

The meniscus is an important component of the knee joint, assisting in load transmission, stress absorption, and joint stability. The meniscus's clinical role in the development of osteoarthritis is well established[29]. A great number of studies have verified the presence of chronic inflammation at the time of meniscal injury (particularly during degenerative meniscal tears) and acute inflammation following traumatic meniscal tears. Degenerative menisci released significantly more matrix-degrading enzymes and inflammatory cytokines. For example, studies examined the gene expression in meniscus tears using QRT-PCR. They found that expression of IL-1 $\beta$, ADAMTS-5, MMP-1, MMP-9, MMP-13, and NFKB was significantly higher in patients with a meniscal tear who were under the age of forty years than it was in those over the age of forty years[30]. Furthermore, when researchers analyzed gene expression in degenerative and traumatic meniscal tears, they discovered that traumatic tears exhibited greater amounts of inflammatory markers, notably chemokines and matrix metalloproteinases, than degenerative tears[31]. Another study found that genes involved in inflammation and cytokine production were up-regulated in OA knee meniscus cells whereas genes involved in DNA repair pathways were downregulated [32]. Furthermore, even in the absence of osteoarthritic alterations in the joint, 43 percent of patients presented with synovial inflammation at the time of arthroscopic meniscectomy 15 weeks after a traumatic meniscal rupture[33]. Similarly, evidence of inflammation was found in synovial samples taken from 80 percent of patients during arthroscopic meniscectomy, independent of tear type[34]. These findings imply that there may be a significant inflammatory response in the knee joint following meniscal injury.

Following a meniscus injury, the inflammatory milieu not only prevents chondrocytes from synthesizing GAG and collagen but also speeds up the deterioration of the cartilage matrix. An inflammatory microenvironment causes abnormal differentiation of mesenchymal stem cells. The incorporation of synthetic cartilage into normal tissues may be hampered by inflammatory stimuli. Furthermore, 
proinflammatory factors have been reported to restrict cartilage-derived progenitor cells (C-PCS) migration, which might limit the efficacy of recruiting endogenous cells and employing cell-free scaffold in situ repair techniques [24]. IL-1 $\beta$ is a key pro-inflammatory cytokine in the progression of OA, acting as an injury factor that triggers the production of inflammatory mediators and MMPs [35]. The NF-KB pathway was also observed to boost the release of cytokines, chemokines, and matrix-degrading enzymes in IL-1-treated human meniscus cells, as well as their catabolic activity [36]. In addition, IL-1 stimulation boosted MMP1 activity, proteoglycan release, and nitric oxide release in porcine meniscus explants [37]. As a result, IL-1 $\beta$ was utilized as an active stimulus in our investigation to replicate the inflammatory milieu in vitro following meniscus damage or stent installation. In MFCs, IL-1 $\beta$ causes inflammation, oxidative stress, apoptosis, and ECM degradation, according to our findings. STS therapy, however, successfully reversed the cancerous effects of IL-1 $\beta$, resulting in improved MFC proliferative activity and contributing to tissue-engineered meniscus maturation (Fig. 4).

TLRs, or toll-like receptors, are a kind of pattern recognition receptor (PRR) found in the innate immune system. TLRs have a key role in arthritis progression[38]. The TLR intracellular domain and the IL-1R intracellular structure constitute the TLR/IL-1R superfamily, which is a homologous double chain. TLR/IL$1 \mathrm{R}$ binds to the ligand and activates IRAK4 by phosphorylating it. IRAK4 that has been phosphorylated recruits TRAF6. TRAF6 then stimulates the MAPK and NF-KB signaling pathways[39]. In osteoarthritis induced by meniscus damage, the MAPK and NF-KB pathways are important. NF-KB plays a critical role in the regulation of several genes involved in the inflammatory response and cell death control[40]. NF-KB signals are verified to be broadly active in the damaged meniscus, and immunohistochemistry identifies NF-KB phosphorylation $[30,41]$. In addition, the activation of pro-inflammatory in meniscus cells elevated the expression of NF-KB [42]. The three mammalian MAPKs (JNK, ERK, and p38) are involved in critical cellular processes such as differentiation, proliferation, and apoptosis[43]. The MAPK pathway is active in the process of cartilage matrix loss caused by MMP overexpression[44]. P-p38 has been found in the damaged meniscus by immunohistochemistry[30]. TLR/IL-1R signaling requires IRAK4 and TRAF6, which play a key role in the start and control of TLR/IL-1R-mediated inflammatory diseases. In osteoarthritis, the expression of IRAK4 and TRAF6 was dramatically enhanced in the synovium and cartilage[39]. In our study, IL-1 $\beta$ markedly induced the phosphorylation of ERK, p38, JNK, and p65. However, these changes could be restored by STS. Further studies showed that STS significantly inhibited il-1 $\beta$-induced upregulation of IRAK4 and TRAF6(Fig. 5).

A three-dimensional structure suited for cell attachment, proliferation, and tissue development, as well as active substances to decrease inflammation and protect meniscus cells from inflammatory damage, is required for the optimal tissue-engineered bionic meniscus scaffold. The knee meniscus is characterized by a complicated structural arrangement of outer and interior regions as heterogeneous fibrocartilage. We retrieved outer MECM and inner MECM, respectively, in this work. Results of safranin 0 and TB staining showed that the inner region was deeply stained, while the outer region was shallow, which was similar to the results of the native meniscus, indicating that outer MECM and inner MECM could well retain native meniscus components, and the differences in regional components were also preserved (Figure S4). As far as we know, this is the first time MECM has been extracted based on area. However, we just employed 
MECM as part of the scaffold building in this experiment and did not perform in-depth research. Previous studies in our laboratory confirmed that $2 \%$ MECM based hydrogel was more conducive to maintaining the activity of MFCs and promoting cell proliferation. In addition,2\% MECM based hydrogel can better promote the secretion of collagen and GAG in MFCs, which means that $2 \%$ MECM based hydrogel can well simulate the microenvironment of MFCs and promote the high expression of specific genes [5]. Therefore, $2 \%$ of MECM was chosen to prepare STS delivery MECM based hydrogel hybrid scaffold.

The tissue structure and mechanical qualities of the regenerated meniscus in the PCL-Hydrogel-MFCSSTS10 group were better than those in the PCL-Hydrogel-MFCs group in vivo experiments, showing the promise of STS for meniscus regeneration. Meniscus regeneration was not seen in the positive control group, suggesting that meniscus regeneration or self-healing capability is severely restricted in the absence of intervention (Fig. 8A). Furthermore, the PCL-hydrogel-MFCS-STS10 group was more successful than the PCL-hydrogel-MFCs group in avoiding cartilage injury (Fig. 8B). This might be because the meniscus protects cartilage surfaces by transporting, stabilizing, and absorbing shocks, and the regenerated meniscus's lower size and biomechanical features result in less cartilage protection.

We also proposed the possible mechanism of the scaffold during meniscus regeneration. For starters, owing to the scaffold's non-specific inflammatory response, a substantial number of M1 macrophages were concentrated around the scaffold at a relatively early stage after scaffold implantation. Second, a large number of STS released from the scaffold in the early stage not only converted M1 macrophages into M2 macrophages in the microenvironment but also prevented MFCs from oxidative stress, apoptosis, and ECM degradation induced by inflammatory stimulation, thus showing stronger proliferation activity. Third, the mixed scaffold degrades slowly in the body. Third, in the body, the mixed scaffold degrades slowly. Finally, their combined efforts resulted in total meniscus regeneration (Fig. 9). There are also some deficiencies in this experiment. First, the early host response and macrophage polarization of the hybrid scaffold were not evaluated in the joint cavity. In addition, degradation of the hybrid scaffold in vivo was not studied. Finally, about three months, the in vivo data were available. As a result, the scaffold should be evaluated over a longer period of time.

\section{Conclusion}

STS delivery PCL-MECM based hydrogel hybrid scaffold promotes meniscal regeneration through controlling the phenotype of macrophage, which provides a new direction for tissue engineering meniscus regeneration.

\section{Methods}

\section{Cell culture}

Air embolization was used to kill 4-week-old New Zealand white rabbits and 8-week-old C57BL/6 mice acquired from Beijing Weitong Lihua Company (Beijing, China). The PLA General Hospital's Independent 
Ethics Committee authorized all animal experimentation techniques. MFCs from mice and rabbits were isolated and cultured using a prior method[4, 5]. Simply put, the meniscus was taken under sterile circumstances, the lateral synovium and lateral $1 / 3$ of the meniscus were removed, and the medial $2 / 3$ of the meniscus was preserved. The tissue was cut and processed for 6 hours with 0.15 percent Type II collagenase. Following that, the cell suspension was centrifuged (1500 $/ \mathrm{min}$ for 5 minutes) to collect the main MFCs. Finally, the separated cells were resuspended in Dulbecco's minimal essential medium (DMEM)/F12 (Corning, US) supplemented with 10\% fetal bovine serum (FBS) and 100U/ml penicillin/streptomycin solution (Gibco, US) at $37^{\circ} \mathrm{C}$ with $5 \% \mathrm{CO}$. Every $2-3$ days, the culture media was replaced. RAW264.7 macrophage cell lines were grown at $37^{\circ} \mathrm{C}$ in humidified conditions with $5 \% \mathrm{CO} 2$ in DMEM (Gibco, US) with 10\% FBS and 100U/ml penicillin/streptomycin solution.

\section{Effects of STS on cell viability}

Effects of STS on cell viabilities were assessed by Cell Counting Kit-8 (CCK-8). RAW264.7 macrophages $\left(1 \times 10^{4 /}\right.$ well $)$ and rabbit MFCs $\left(5 \times 10^{3 /}\right.$ well $)$ were seeded in 96 -well plates. Cells were treated with various concentrations of STS $(0,5,10,20,40 \mathrm{ug} / \mathrm{ml})$ for $24 \mathrm{~h}$. Afterward, $100 \mu \mathrm{l}$ complete culture medium containing $10 \mu \mathrm{l} \mathrm{CCK-8} \mathrm{solution} \mathrm{(Dojindo,} \mathrm{Japan)} \mathrm{was} \mathrm{added} \mathrm{to} \mathrm{each} \mathrm{well} \mathrm{and} \mathrm{incubated} \mathrm{at} 37^{\circ} \mathrm{C}$ in darkness for $4 \mathrm{~h}$. The absorbance at $450 \mathrm{~nm}$ of each well was detected using a microplate reader (Beckman, USA).

\section{Effects of STS on macrophage repolarization}

RAW264.7 macrophages were primed with 100ng/ml LPS (Sigma, US) for 24 hours to exhibit the M1 phenotype in order to assess macrophage repolarization from M1 to M2. To achieve the M2 phenotype, $20 \mathrm{ng} / \mathrm{mL}$ interleukin (IL-4) (PeproTech, US) was utilized. Following that, the M1 macrophages were treated with varying dosages of STS for an additional 24 hours. The polarization transitions were studied using quantitative real-time polymerase chain reaction (qRT-PCR), enzyme-linked immunosorbent assay (ELISA), and immunofluorescent (IF) labeling.

\section{RAW264.7 mouse macrophages conditioned medium (CM) collection and its effect on mouse MFCs.}

For 24 hours, RAW264.7 macrophages were stimulated with LPS to show the M1 phenotype and IL-4 to show the M2 phenotype. For an additional 24 hours, the M1 macrophages were exposed to different doses of STS. The supernatants of macrophages were extracted and centrifuged at $1000 \mathrm{~g}$ for 5 minutes before being stored at $-80^{\circ} \mathrm{C}$ for future research. The $\mathrm{CM}$ from developed macrophages was diluted in serum-free media at a 1:1 ratio before being introduced to mouse MFCs for chondrocyte apoptosis investigation. 


\section{Effects of STS on IL-1 $\beta$-induced inflammation, oxidative stress, apoptosis, and ECM degradation in rabbit MFCs.}

Rabbit MFCs were treated with or without $10 \mathrm{ng} / \mathrm{mL} \mathrm{IL}-1 \beta$ and various concentrations of STS (10, $20 \mathrm{ug} / \mathrm{ml}$ ) for $24 \mathrm{~h}$. The effects of STS were evaluated by qRT-PCR, ELISA, western blot, and IF staining.

\section{Quantitative real-time PCR (qRT-PCR)}

TRIzol(Ambion, USA) was used to extract total RNA from rabbit MFCs and RAW264.7 macrophages. A spectrophotometer was used to determine the concentration and purity of the isolated RNA. The RNA was then reverse-transcribed, and the resulting cDNA was amplified using polymerase chain reaction (PCR). PCR experiments (per well: 1 uL CDNA, 0.5 uL forward and reverse primers, 10 uL 2RealStar Green Fast Mixture (with ROX), 8 uL ddH2O) were carried out using an SYBR Green real-time PCR kit (GenStar) and the ArchimedX6 qRT-PCR system. All studies were repeated at least three times, and the amplification signals from individual target genes were standardized to glyceraldehyde3-phosphate dehydrogenase (GAPDH) levels. The primer sequences were shown in Table S1.

\section{ELISA}

For RAW264.7 macrophages, the levels of IL-1 $\beta$ and TNF-a were evaluated using the mouse IL-1 $\beta$ ELISA kit (mlbio, ml301814), mouse TNF-a ELISA kit (mlbio, ml002095-J). For rabbit MFCs, rabbit MFCs were treated with IL-1 $\beta(10 \mathrm{ng} / \mathrm{ml})$ in the presence or absence of different concentrations (10 and $20 \mu \mathrm{g} / \mathrm{ml})$ of STS for $24 \mathrm{~h}$. The culture supernatants were harvested for the following measurement. The levels of IL-1 $\beta$, TNF-a, and matrix metalloproteinase13(MMP-13) were evaluated using the rabbit IL-1 $\beta$ ELISA kit (Jianglai, E72059), rabbit TNF-a ELISA kit (Jianglai, E72292), and rabbit MMP-13 ELISA kit (Jianglai, E72146). The measurement was performed in line with the product's instruction booklet.

\section{Immunofluorescent staining}

RAW264.7 macrophages and rabbit MFCs were fixed in paraformaldehyde with 0.1\% Triton X-100 (Sigma, USA). To inhibit nonspecific binding, 10\% goat serum was utilized. The cells were then treated with antibodies overnight. The cells were treated for 1 hour at room temperature with fluorophore-conjugated secondary antibodies (Alexa Fluor goat anti-mouse 488 or goat anti-rabbit 488, Abcam) at a 1:100 dilution to visualize the relevant subsets. Cells were then stained with 4,6-diamidino-2-phenylindole (DAPI) for 5 minutes. The stained slides were examined under a fluorescence microscope (Nikon, Tokyo). Image $\mathrm{J}$ (version 1.5.0, USA) was used to count the number of positive cells. The following antibodies are used: inos(Abcam). CD206(CST). ACAN(Novus). and collagen II (Novus).

\section{Flow cytometry for mouse MFCs apoptosis analysis}

Mouse MFCs $\left(10^{6} /\right.$ well) in a 6-well plate were treated with collected RAW264.7 mouse macrophages conditioned medium for 3 days. Mouse MFCs apoptosis rates were analyzed with annexin V-FITC 
apoptosis detection kit (Beyotime) according to the manufacturer's instructions.

\section{Western blot}

In the icebox, rabbit MFCs were lysed for $1.5 \mathrm{~h}$ with RIPA lysate containing 0.1 percent PMSF. The fractured samples were then spun at 12,000 RPM for 20 minutes at $4^{\circ} \mathrm{C}$ in a centrifuge. The supernatant is the complete protein solution. The bicinchoninic acid technique was used to determine protein concentration and compute loading volume. The separating gel and concentrating gel were then prepared, and electrophoresis commenced after loading samples in a certain order. After electrophoresis on the gel substrate, the gel was removed and transferred to the PVDF membrane. The membrane and the first antibody $(1: 1000)$ were incubated overnight at $4^{\circ} \mathrm{C}$. The membrane was incubated with the second antibody (1:3000) at room temperature for 1 hour on the second day. Finally, the gray value is determined after the PVDF membrane has been exposed to a $200 \mathrm{uL} E C L$ solution. The following antibodies are used: Interleukin-1 receptor-associated kinase 4(IRAK4, CST), TNFR-associated factor 6 (TRAF6, CST), p-IкB (Bioss), nuclear factor kappa-B (p-NFkB p65, Bioss), extracellular regulated protein kinases (p-ERK, Bioss), p-p38 (Bioss), c-Jun N-terminal kinase (p-JNK, Bioss), and $\beta$-actin (Abcam).

\section{Fabrication of the scaffold}

\section{Fabrication of STS delivery MECM based hydrogel hybrid solutions}

The method for preparing and identifying inner MECM and outer MECM are shown in the Supplementary Material. STS delivery MECM based hydrogel hybrid solutions were made by dissolving sodium alginate in $10 \mathrm{ug} / \mathrm{ml}$ STS solution at $2 \%(\mathrm{w} / \mathrm{v})$ containing $2 \%$ MECM particles.

\section{Fabrication of STS delivery PCL-MECM based hydrogel hybrid scaffold}

The rabbit meniscus was completely removed from the knee joint. Micro-ct was used to obtain imaging information of the native meniscus. Solidworks 2018 software was used to design a pure PCL scaffold model and export it in STL format. The scaffold was then fabricated by a 3D layer-by-layer fused deposition modeling (FDM) printer (PanoSpace BioPro) using PCL (Mn = 80000, Sigma) (Table S2). Inner MECM based hydrogel hybrid Solutions and outer MECM based hydrogel hybrid solutions of sustainedrelease STS were injected into the inner and outer sides of the PCL scaffold respectively. After that, the hybrid scaffold was cross-linked for 2 minutes using $102 \mathrm{mM} \mathrm{CaCl} 2$ ions.

\section{STS releasing behavior of the hybrid scaffold}

In order to measure the STS releasing behavior of the MECM based hydrogel, STS delivery MECM based hydrogel hybrid solutions were poured into a $3 \mathrm{~mm}$ diameter mold, then crosslinked with $102 \mathrm{mM} \mathrm{CaCl} 2$ ions for 2 min. scaffolds were immersed in PBS at $37^{\circ} \mathrm{C}$ during the test period. At each time point $(1 \mathrm{~h}, 3$ h, 6 h, 12 h, 12 h, 18 h, 24 h, 30 h, 36 h, 42 h, and 48 h), $200 \mathrm{ul}$ of supernatant was removed and replaced with an equal volume of fresh PBS. Then the absorbance of supernatants at $265 \mathrm{~nm}$ wavelength was determined by UV spectrophotometer and the concentration of STS in the supernatants was calculated according to the standard curve of STS. 


\section{Cell viability analysis}

To assess the biocompatibility of the scaffolds, a Live Death@ Viability/Cytotoxicity Kit (BioVision, San Francisco, America) was employed. MFCs were added at a density of $5 \times 10^{5} / \mathrm{mL}$ to STS delivery MECMbased hydrogel hybrid solutions before the fabrication of an MFCs-loaded hybrid scaffold. The MFCsloaded hybrid scaffold was cultured for three days before being stained with live-dead staining according to the product manual. Then, we imaged at 488 and $552 \mathrm{~nm}$ with a Leica TCS-SP8 laser confocal microscope (Wetzlar, Germany).

\section{In vivo assessment of the early host response and macrophage polarization of the hybrid scaffold.}

Eight Sprague Dawley (SD) rats were used to evaluate the early host response and macrophage polarization of the hybrid scaffolds in vivo. Eight Sprague Dawley (SD) rats were randomly divided into two groups of four rats each. They were PCL-MECM based hydrogel hybrid scaffold (PCL-hydrogel) and STS delivery PCL-MECM based hydrogel hybrid scaffold (PCL-hydrogel-STS10). Following anesthesia, the hybrid scaffold is inserted into the subcutaneous tissue. The rats were killed one week after implantation. Remove the scaffold, along with the surrounding capsule and tissue. Frozen microtomes were used to cut the samples into 5um thick pieces after OCT embedding was completed. The levels of inflammation and macrophage polarization were assessed by hematoxylin and eosin (H\&E), immunofluorescence staining (CD206, CST), and immunohistochemical staining (IL-1 $\beta$, Abcam). For immunohistochemical staining, sections were treated with the first antibodies for an overnight period at $4^{\circ} \mathrm{C}$. Sections were then incubated in the second antibody for 1 hour after being rinsed three times with PBS. After being sealed with neutral resins, the portions were exposed to DAB solution and photographed. Dark brown is considered a positive expression. Image $\mathrm{J}$ software (version 1.5.0, USA) was used for quantitative/semiquantitative analysis of the results.

\section{In vivo repair}

\section{Surgical procedure}

A total of twenty New Zealand white rabbits (male, five months old) were obtained from the Beijing Weitong Lihua Company (Beijing, China). At the PLA General Hospital, the study was approved by the Institutional Animal Care and Use Committee. Twenty rabbits were randomly divided into four groups of five rabbits each (two knees of each rabbit were used). They were the negative control group (negative control), PCL-MECM based hydrogel hybrid scaffold group (PCL-hydrogel-MFCs), STS delivery hybrid scaffold group (PCL-hydrogel-MFCs-STS10), and the positive control group (positive control).

The method for establishing critical-size medial meniscal defect was performed as described in our previous study $[4,45]$. Briefly, To expose the internal structure of the knee joint, the patella is rotated laterally. The rabbits were then subjected to an entire medial meniscectomy, with the exception of $5 \%$ of the external rim, which was implanted with a scaffold and sutured to the residual rim. MFCs were added into hybrid scaffold at a density of $5 \times 10^{6} / \mathrm{mL}$ in the PCL-Hydrogel-MFCs groups and PCL-Hydrogel- 
MFCS-STS10 groups. Culture in DMEM for 24h one day before transplantation. The rabbits in the negative control group had a fake procedure, whereas the rabbits in the positive control group merely had meniscectomy. Layer by layer, the wound was sutured. The rabbits were maintained in fixed, individual cages after surgery and were thoroughly monitored for infection and other problems. Air embolization killed rabbits three months later. Meniscus and cartilage samples from the knee were obtained for further examination.

\section{Histological analysis}

After the animals were killed, the meniscus was fixed in $4 \%$ paraformaldehyde for $2 \mathrm{~d}$, paraffin embedding, and cut at approximately $7 \mu \mathrm{m}$. Following the manufacturer's instructions, the sections were stained with $\mathrm{H} \& \mathrm{E}$ and toluidine blue (TB). The meniscal regeneration was then estimated using a semiquantitative histological grading method. Three independently trained researchers blindly analyzed all photos in terms of reparative tissue with bonding, the presence of fibrochondrocytes, and safranin 0 stainability using the Ishida scoring system[46]. The articular surface was examined by fixing osteochondral samples from the femur and the tibia for 48 hours in 4 percent paraformaldehyde and then decalcifying for 7 weeks in EDTA solution. the samples were sliced into 7um slices and stained with H\&E. An individual specimen's score was based on the mean value from all of its cartilage sections, according to Mankin scoring criteria[47].

\section{Statistical analysis}

All data were expressed as means \pm standard deviation (SD) for a minimum of $n=3$. SPSS 22.0 statistical software was used for the statistical analysis. Statistical significance was indicated by a pvalue $<0.05$. T-tests were used to compare the two groups. One-way ANOVA was used to compare the three groups.

\section{Declarations}

Acknowledgments: no

\section{Authors' contributions}

SL, ZC, and QG conceived and designed the study, ML, HY, and MC performed experiments; $M L, H Y$ analyzed the data; QG, ZC, MC, XS, HD, CN, ZY, GT, and ZY provided advice and technical assistance; and $M L, H Y$ wrote the manuscript. All authors read and approved the final manuscript.

\section{Funding}

This work was supported by the National Natural Science Foundation of China (81972070), PLA Youth Project for Medical Science (18QNP057), the key project under the guidance of Hunan Provincial Health Commission (20200476, 20201907), and the Scientific research Project of Hunan Provincial Health Commission (202102080160). 
Availabability of data and materials

All data generated or analyzed during this study are included in this article.

\section{Ethics approval and consent to participate}

All of the experimental procedures involving animals were conducted in accordance with protocol approved by Institutional Animal Care and Use Committee of PLA General Hospital, Beijing, China(S2020046-01)

\section{Consent for publication}

All authors agree to be published.

\section{Competing interests}

All authors declared no conflict of interest.

\section{Authors' information (optional)}

1 Institute of Orthopedics, The First Medical Center, Chinese PLA General Hospital, Beijing Key Lab of Regenerative Medicine in Orthopedics, Key Laboratory of Musculoskeletal Trauma and War Injuries PLA, No. 28 Fuxing Road, Haidian District, Beijing 100853, China

2 Department of Orthopedics, The First Affiliated Hospital, Hengyang medical school, University of South China, Hengyang 421000, China

3 Department of Orthopedic Surgery, Beijing Jishuitan Hospital, Fourth Clinical College of Peking University, Beijing 100035, China

${ }^{4}$ Department of Bone and Joint Surgery, Renji Hospital, School of Medicine, Shanghai Jiaotong University, Shanghai 200030, China.

\section{References}

1. Makris EA, Hadidi P, Athanasiou KA: The knee meniscus: structure-function, pathophysiology, current repair techniques, and prospects for regeneration. Biomaterials 2011, 32:7411-7431.

2. Englund M, Roemer FW, Hayashi D, Crema MD, Guermazi A: Meniscus pathology, osteoarthritis and the treatment controversy. Nat Rev Rheumatol 2012, 8:412-419.

3. Wang X, Ding Y, Li H, Mo X, Wu J: Advances in electrospun scaffolds for meniscus tissue engineering and regeneration. J Biomed Mater Res B Appl Biomater 2021.

4. Guo W, Chen M, Wang Z, Tian Y, Zheng J, Gao S, Li Y, Zheng Y, Li X, Huang J, et al: 3D-printed cell-free PCL-MECM scaffold with biomimetic micro-structure and micro-environment to enhance in situ 
meniscus regeneration. Bioact Mater 2021, 6:3620-3633.

5. Chen M, Feng Z, Guo W, Yang D, Gao S, Li Y, Shen S, Yuan Z, Huang B, Zhang Y, et al: PCL-MECMBased Hydrogel Hybrid Scaffolds and Meniscal Fibrochondrocytes Promote Whole Meniscus Regeneration in a Rabbit Meniscectomy Model. ACS Appl Mater Interfaces 2019, 11:41626-41639.

6. Zhang ZZ, Chen YR, Wang SJ, Zhao F, Wang XG, Yang F, Shi JJ, Ge ZG, Ding WY, Yang YC, et al: Orchestrated biomechanical, structural, and biochemical stimuli for engineering anisotropic meniscus. Sci Transl Med 2019, 11.

7. Zhou ZY, Zhao WR, Zhang J, Chen XL, Tang JY: Sodium tanshinone IIA sulfonate: A review of pharmacological activity and pharmacokinetics. Biomed Pharmacother 2019, 118:109362.

8. Du H, Wang Y, Zeng Y, Huang X, Liu D, Ye L, Li Y, Chen X, Liu T, Li H, et al: Tanshinone IIA Suppresses Proliferation and Inflammatory Cytokine Production of Synovial Fibroblasts from Rheumatoid Arthritis Patients Induced by TNF-alpha and Attenuates the Inflammatory Response in AIA Mice. Front Pharmacol 2020, 11:568.

9. Feng M, Peng H, Yao R, Zhang Z, Mao G, Yu H, Qiu Y: Inhibition of cellular communication network factor 1 (CCN1)-driven senescence slows down cartilage inflammaging and osteoarthritis. Bone 2020, 139:115522.

10. Wang X, Fan J, Ding X, Sun Y, Cui Z, Liu W: Tanshinone I Inhibits IL-1beta-Induced Apoptosis, Inflammation And Extracellular Matrix Degradation In Chondrocytes CHON-001 Cells And Attenuates Murine Osteoarthritis. Drug Des Devel Ther 2019, 13:3559-3568.

11. Jia PT, Zhang XL, Zuo HN, Lu X, Li L: Articular cartilage degradation is prevented by tanshinone IIA through inhibiting apoptosis and the expression of inflammatory cytokines. Mol Med Rep 2017, 16:6285-6289.

12. Zhang S, Huang G, Yuan K, Zhu Q, Sheng H, Yu R, Luo G, Xu A: Tanshinone IIA ameliorates chronic arthritis in mice by modulating neutrophil activities. Clin Exp Immunol 2017, 190:29-39.

13. Zhang Y, Sun L, Liu X, Zhu D, Dang J, Xue Y, Fan H: Investigating the protective effect of tanshinone IIA against chondrocyte dedifferentiation: a combined molecular biology and network pharmacology approach. Ann Transl Med 2021, 9:249.

14. Chen W, Xu Y, Li H, Dai Y, Zhou G, Zhou Z, Xia H, Liu H: Tanshinone IIA Delivery Silk Fibroin Scaffolds Significantly Enhance Articular Cartilage Defect Repairing via Promoting Cartilage Regeneration. ACS Appl Mater Interfaces 2020, 12:21470-21480.

15. Klimak M, Nims RJ, Pferdehirt L, Collins KH, Harasymowicz NS, Oswald SJ, Setton LA, Guilak F: Immunoengineering the next generation of arthritis therapies. Acta Biomater 2021, 133:74-86.

16. Fernandes TL, Gomoll AH, Lattermann C, Hernandez AJ, Bueno DF, Amano MT: Macrophage: A Potential Target on Cartilage Regeneration. Front Immunol 2020, 11:111.

17. Brown BN, Ratner BD, Goodman SB, Amar S, Badylak SF: Macrophage polarization: an opportunity for improved outcomes in biomaterials and regenerative medicine. Biomaterials 2012, 33:37923802. 
18. Xie J, Huang Z, Yu X, Zhou L, Pei F: Clinical implications of macrophage dysfunction in the development of osteoarthritis of the knee. Cytokine Growth Factor Rev 2019, 46:36-44.

19. Kiraz Y, Adan A, Kartal Yandim M, Baran Y: Major apoptotic mechanisms and genes involved in apoptosis. Tumour Biol 2016, 37:8471-8486.

20. Chen L, Zheng L, Chen P, Liang G: Myeloid Differentiation Primary Response Protein 88 (MyD88): The Central Hub of TLR/IL-1R Signaling. J Med Chem 2020, 63:13316-13329.

21. Nakkala JR, Li Z, Ahmad W, Wang K, Gao C: Immunomodulatory biomaterials and their application in therapies for chronic inflammation-related diseases. Acta Biomater 2021, 123:1-30.

22. Fujihara Y, Abe T, Hoshi K: Controlling the Phenotype of Macrophages Promotes Maturation of Tissue-Engineered Cartilage. Tissue Eng Part A 2020, 26:1005-1013.

23. Schmidt-Bleek K, Kwee BJ, Mooney DJ, Duda GN: Boon and Bane of Inflammation in Bone Tissue Regeneration and Its Link with Angiogenesis. Tissue Eng Part B Rev 2015, 21:354-364.

24. Li M, Yin H, Yan Z, Li H, Wu J, Wang Y, Wei F, Tian G, Ning C, Li H, et al: The immune microenvironment in cartilage injury and repair. Acta Biomater 2021.

25. Tian G, Jiang S, Li J, Wei F, Li X, Ding Y, Yang Z, Sun Z, Zha K, Wang F, et al: Cell-free decellularized cartilage extracellular matrix scaffolds combined with interleukin 4 promote osteochondral repair through immunomodulatory macrophages: In vitro and in vivo preclinical study. Acta Biomater 2021, 127:131-145.

26. Rees AJ: Monocyte and macrophage biology: an overview. Semin Nephrol 2010, 30:216-233.

27. Im GI: Biomaterials in orthopaedics: the past and future with immune modulation. Biomater Res 2020, $24: 7$.

28. Badylak SF, Valentin JE, Ravindra AK, McCabe GP, Stewart-Akers AM: Macrophage phenotype as a determinant of biologic scaffold remodeling. Tissue Eng Part A 2008, 14:1835-1842.

29. Englund M, Felson DT, Guermazi A, Roemer FW, Wang K, Crema MD, Lynch JA, Sharma L, Segal NA, Lewis CE, Nevitt MC: Risk factors for medial meniscal pathology on knee MRI in older US adults: a multicentre prospective cohort study. Ann Rheum Dis 2011, 70:1733-1739.

30. Brophy RH, Rai MF, Zhang Z, Torgomyan A, Sandell LJ: Molecular analysis of age and sex-related gene expression in meniscal tears with and without a concomitant anterior cruciate ligament tear. $J$ Bone Joint Surg Am 2012, 94:385-393.

31. Brophy RH, Sandell LJ, Rai MF: Traumatic and Degenerative Meniscus Tears Have Different Gene Expression Signatures. Am J Sports Med 2017, 45:114-120.

32. Sun Y, Mauerhan DR, Honeycutt PR, Kneisl JS, Norton JH, Hanley EN, Jr., Gruber HE: Analysis of meniscal degeneration and meniscal gene expression. BMC Musculoskelet Disord 2010, 11:19.

33. Scanzello CR, McKeon B, Swaim BH, DiCarlo E, Asomugha EU, Kanda V, Nair A, Lee DM, Richmond $\mathrm{JC}$, Katz JN, et al: Synovial inflammation in patients undergoing arthroscopic meniscectomy: molecular characterization and relationship to symptoms. Arthritis Rheum 2011, 63:391-400. 
34. Cantatore FP, Benazzo F, Ribatti D, Lapadula G, D'Amico S, Tursi A, Pipitone V: Early alteration of synovial membrane in osteoarthrosis. Clin Rheumatol 1988, 7:214-219.

35. Kobayashi M, Squires GR, Mousa A, Tanzer M, Zukor DJ, Antoniou J, Feige U, Poole AR: Role of interleukin-1 and tumor necrosis factor alpha in matrix degradation of human osteoarthritic cartilage. Arthritis Rheum 2005, 52:128-135.

36. Loeser RF, Olex AL, McNulty MA, Carlson CS, Callahan MF, Ferguson CM, Chou J, Leng X, Fetrow JS: Microarray analysis reveals age-related differences in gene expression during the development of osteoarthritis in mice. Arthritis Rheum 2012, 64:705-717.

37. McNulty AL, Miller MR, O'Connor SK, Guilak F: The effects of adipokines on cartilage and meniscus catabolism. Connect Tissue Res 2011, 52:523-533.

38. Jenei-LanzI Z, Meurer A, Zaucke F: Interleukin-1 beta signaling in osteoarthritis - chondrocytes in focus. Cell Signal 2019, 53:212-223.

39. Li M, Li H, Ran X, Yin H, Luo X, Chen Z: Effects of adenovirus-mediated knockdown of IRAK4 on synovitis in the osteoarthritis rabbit model. Arthritis Res Ther 2021, 23:294.

40. Lee HW, Ahn DH, Crawley SC, Li JD, Gum JR, Jr., Basbaum CB, Fan NQ, Szymkowski DE, Han SY, Lee $\mathrm{BH}$, et al: Phorbol 12-myristate 13-acetate up-regulates the transcription of MUC2 intestinal mucin via Ras, ERK, and NF-kappa B. J Biol Chem 2002, 277:32624-32631.

41. Papachristou DJ, Papadakou E, Basdra EK, Baltopoulos P, Panagiotopoulos E, Papavassiliou AG: Involvement of the p38 MAPK-NF-kappaB signal transduction pathway and COX-2 in the pathobiology of meniscus degeneration in humans. Mol Med 2008, 14:160-166.

42. Stone AV, Loeser RF, Vanderman KS, Long DL, Clark SC, Ferguson CM: Pro-inflammatory stimulation of meniscus cells increases production of matrix metalloproteinases and additional catabolic factors involved in osteoarthritis pathogenesis. Osteoarthritis Cartilage 2014, 22:264-274.

43. Chang L, Karin M: Mammalian MAP kinase signalling cascades. Nature 2001, 410:37-40.

44. Sondergaard BC, Schultz N, Madsen SH, Bay-Jensen AC, Kassem M, Karsdal MA: MAPKs are essential upstream signaling pathways in proteolytic cartilage degradation-divergence in pathways leading to aggrecanase and MMP-mediated articular cartilage degradation. Osteoarthritis Cartilage 2010, 18:279-288.

45. Yuan Z, Liu S, Hao C, Guo W, Gao S, Wang M, Chen M, Sun Z, Xu Y, Wang Y, et al: AMECM/DCB scaffold prompts successful total meniscus reconstruction in a rabbit total meniscectomy model. Biomaterials 2016, 111:13-26.

46. Ishida K, Kuroda R, Miwa M, Tabata Y, Hokugo A, Kawamoto T, Sasaki K, Doita M, Kurosaka M: The regenerative effects of platelet-rich plasma on meniscal cells in vitro and its in vivo application with biodegradable gelatin hydrogel. Tissue Eng 2007, 13:1103-1112.

47. Mankin HJ, Johnson ME, Lippiello L: Biochemical and metabolic abnormalities in articular cartilage from osteoarthritic human hips. III. Distribution and metabolism of amino sugar-containing macromolecules. J Bone Joint Surg Am 1981, 63:131-139. 
Figures

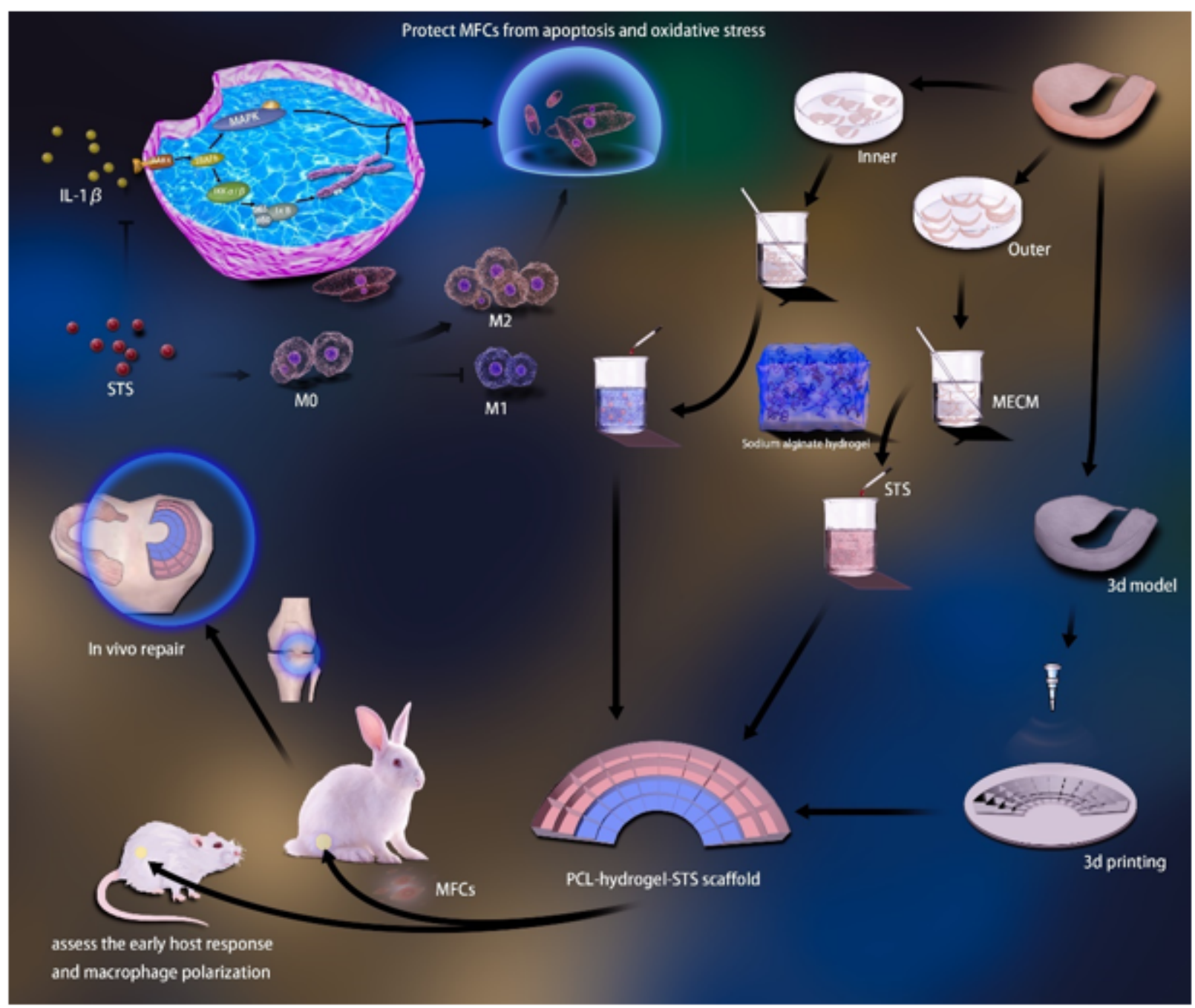

Figure 1

Schematic diagram of the overall study design. 
A

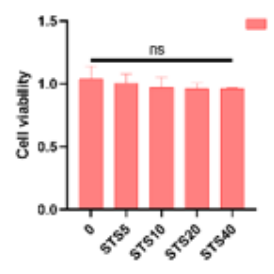

B
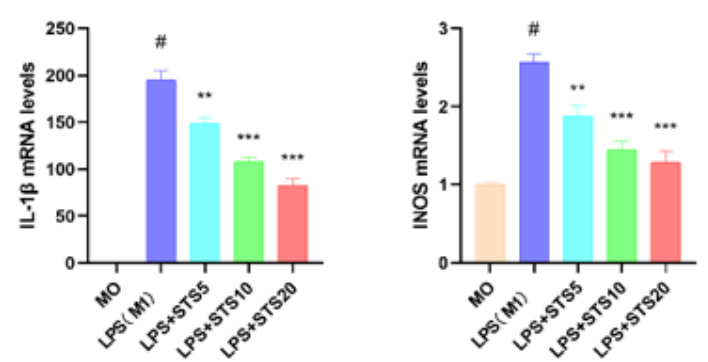

C
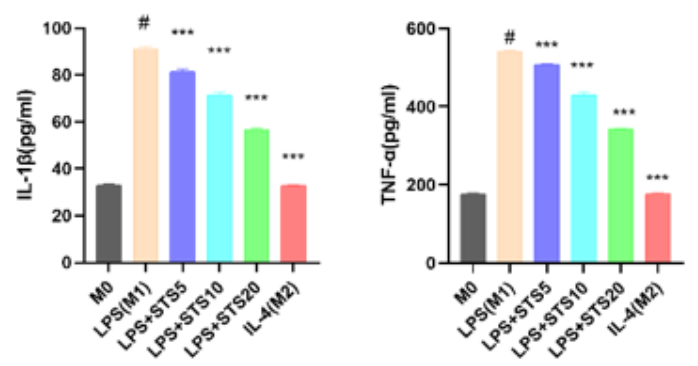
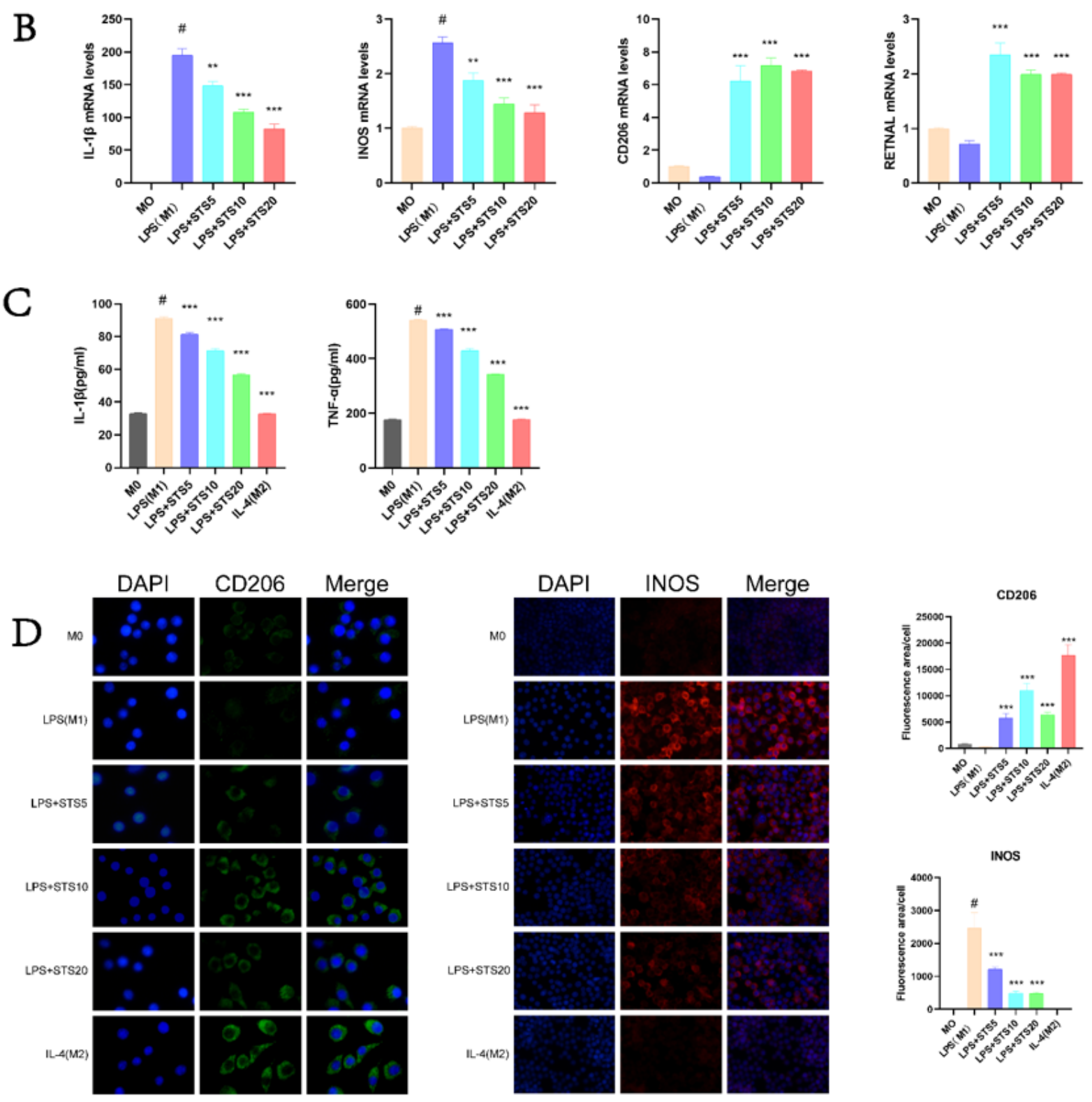

INOS

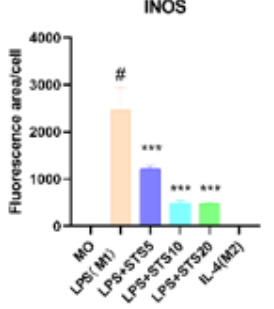

\section{Figure 2}

STS shifts macrophages from M1 to M2 polarization. (A)RAW264.7 macrophages and rabbit MFCs were exposed to STS. Cell viability was determined by the CCK-8 assay. ( $* P<0.05$ vs. 0 group). (B) IL-1 $\beta$, iNOS, CD206, and Rental mRNA expression in RAW264.7 mouse macrophages was detected by qRT-PCR. (C) ELISA was used to detect the level of IL-1 $\beta$ and TNF-a in the supernatant of RAW264.7 macrophages. (D) M1 macrophage marker iNOS and M2 macrophage marker CD206 were examined by 
immunostaining. Data are expressed as the mean \pm SD. Statistical analysis was performed by one-way ANOVA, ${ }^{\#} \mathrm{P}<0.05$ vs. M0 group. ${ }^{*} \mathrm{P}<0.05,{ }^{\star *} \mathrm{P}<0.01,{ }^{\star \star *} \mathrm{P}<0.001$ vs. LPS(M1) group. $\mathrm{n}=3$ per group.

A
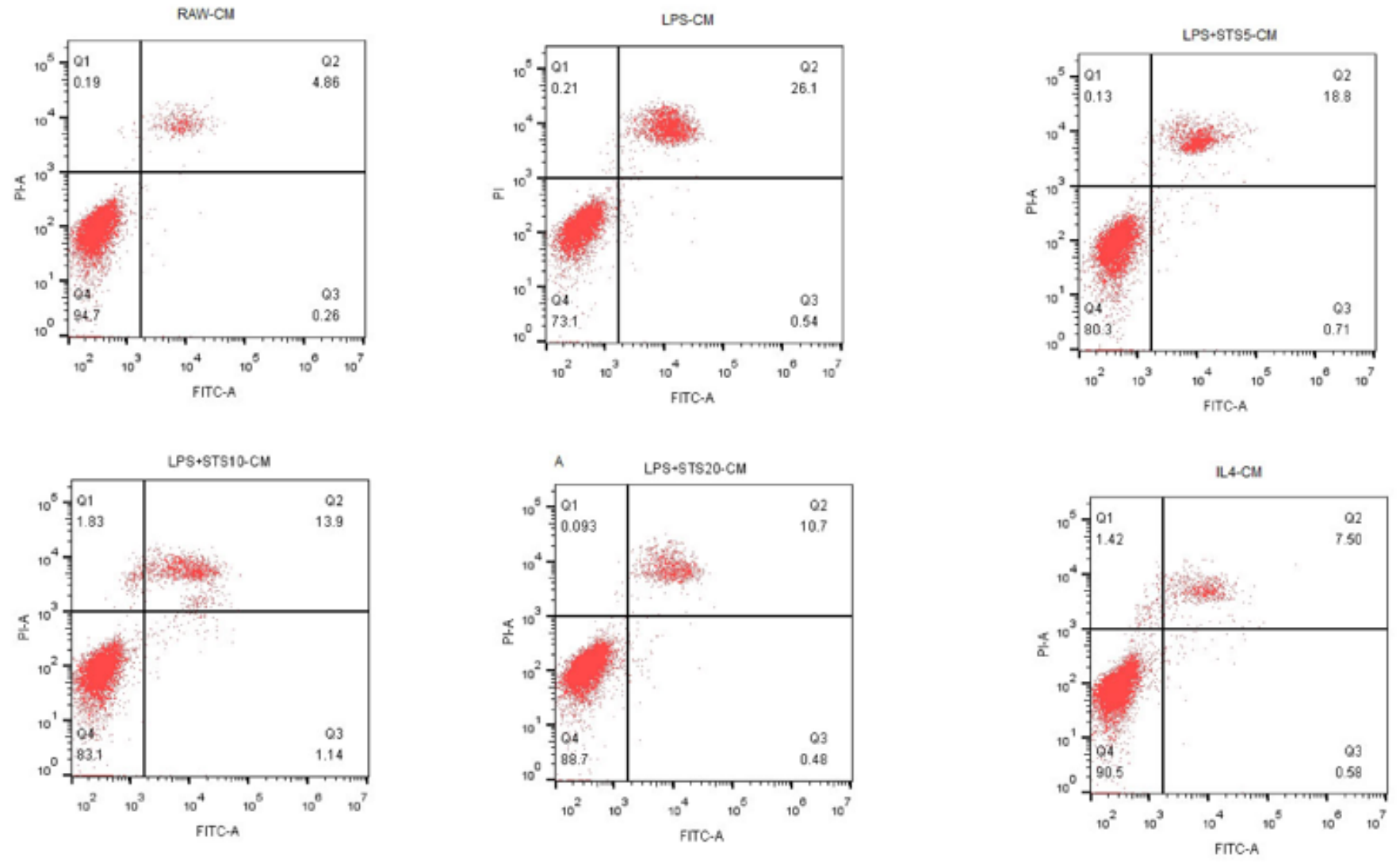

B

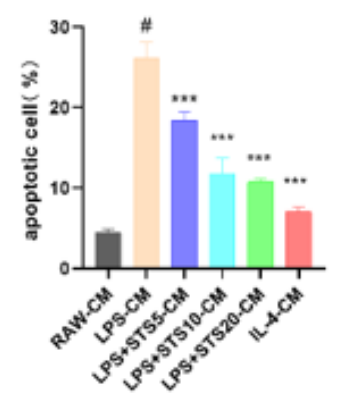

\section{Figure 3}

STS protects MFCs against the effects of macrophage $C M$. (A, B) Apoptosis rates for macrophage CMstimulated MFCs were analyzed by flow cytometry with annexin V-FITC/PI apoptosis analysis. Data are expressed as the mean \pm SD. Statistical analysis was performed by one-way ANOVA, ${ }^{*} \mathrm{P}<0.05$ vs. M0-CM group. ${ }^{*} * \mathrm{P}<0.001 \mathrm{vs}$. LPS-CM group. $\mathrm{n}=3$ per group. 
A
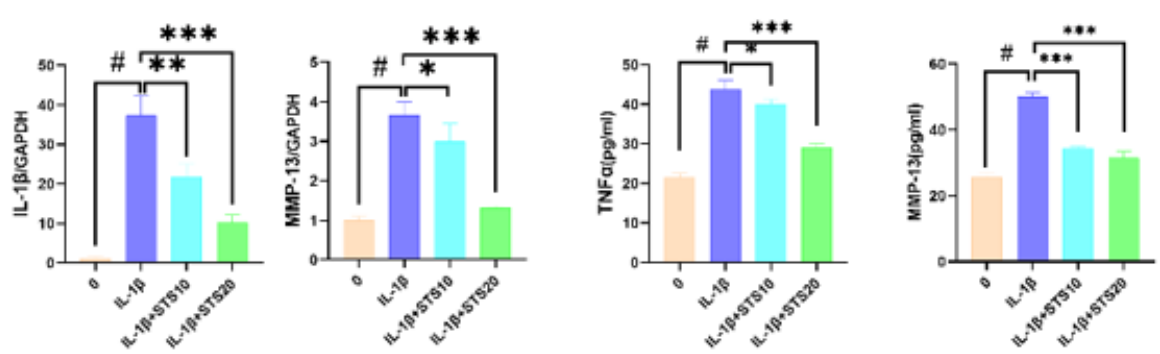

B
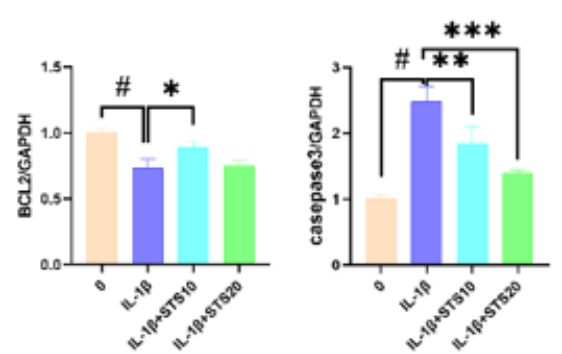

C

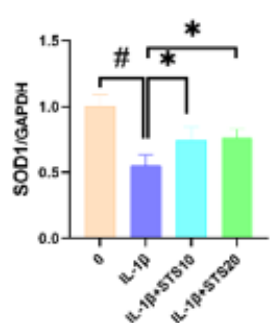

$\mathrm{D}$
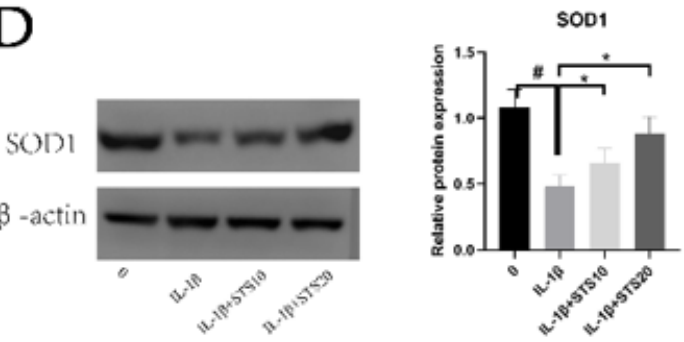

E

$\mathrm{IL}-1 \beta$
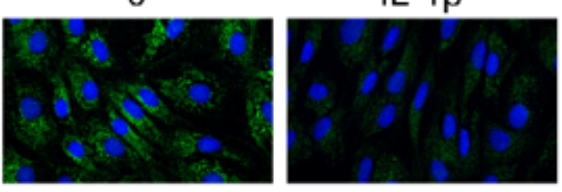

IL-1 $\beta+$ STS10

ACAN

Collagen II
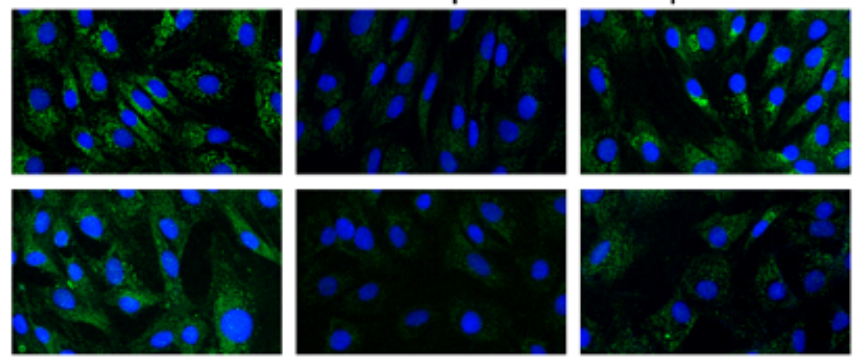

IL-1 $1 \beta+S T S 20$
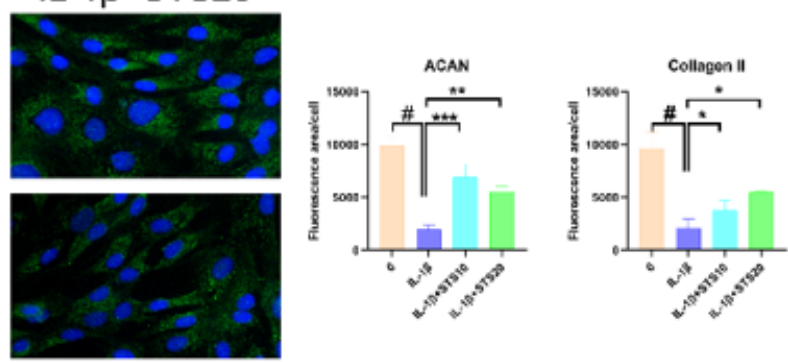

Figure 4

STS protects MFCs from IL-1 $\beta$-induced inflammation, oxidative damage, apoptosis, and ECM breakdown. (A) The levels of IL-1 $\beta$, TNF- $\alpha$, and MMP-13 in MFCs were detected by qRT-PCR and ELISA. (B) Bcl-2 and Caspase-3, two apoptosis-related key genes, were chosen to investigate the effects of STS on apoptosis of MFCs by qRT-PCR. (C-D) The mRNA and protein expression of SOD1 was detected by western blotting and qRT-PCR. (E) Collagen II and ACAN were observed by immunofluorescence after MFCs were treated with IL-1 $\beta(10 \mathrm{ng} / \mathrm{ml})$ with or without STS $(10 \mathrm{ug} / \mathrm{ml}, 20 \mathrm{ug} / \mathrm{ml})$. Data are expressed as the mean $\pm S D$. Statistical analysis was performed by one-way ANOVA, ${ }^{\#} P<0.05$ vs. 0 groups. ${ }^{*} P<0.05$, $\star * P<0.01, * \star * P<0.001$ vs. IL-1 $\beta$ group. $n=3$ per group. 
A

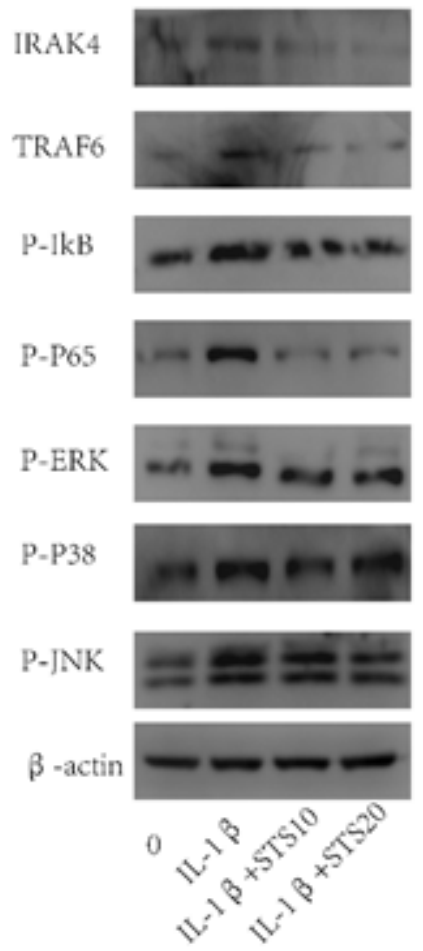

B
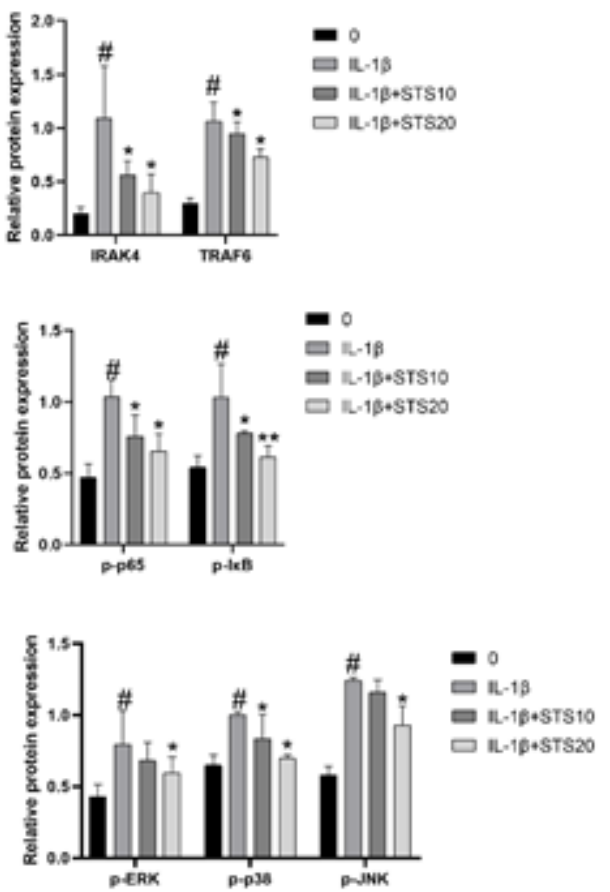

\section{Figure 5}

Effects of STS on IRAK4/TRAF6/NFKB signaling pathway. (A) Protein levels of IRAK4, TRAF6, p-IKB, pp65, p-JNK, p-ERK, and p-p38 were detected by Western blot. (B) Relative protein expression was quantified by ImageJ software. Data are expressed as the mean \pm SD. Statistical analysis was performed by one-way ANOVA, ${ }^{\#} \mathrm{P}<0.05$ vs. 0 groups. ${ }^{*} \mathrm{P}<0.05,{ }^{*} \mathrm{P}<0.01$ vs. IL-1 $\beta$ group. $\mathrm{n}=3$ per group. 
A

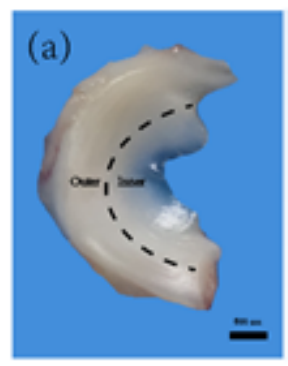

(b)

C
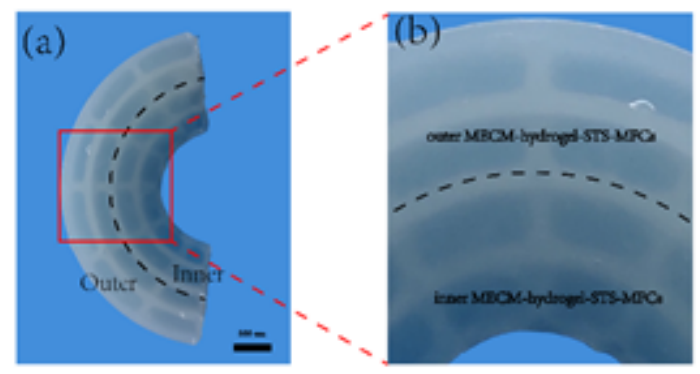

E

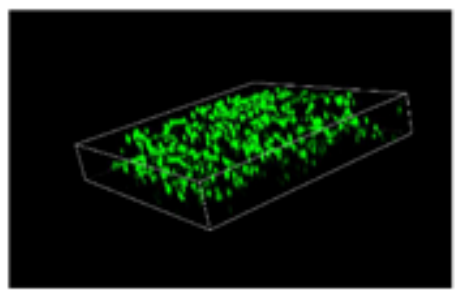

Live

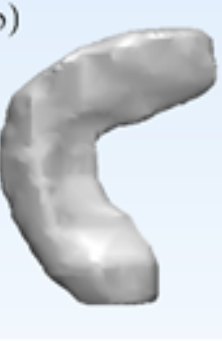

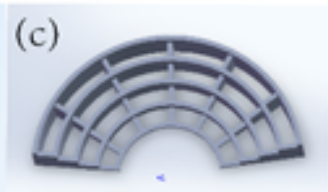

(d)

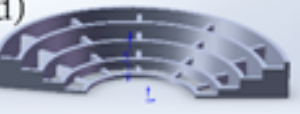

B

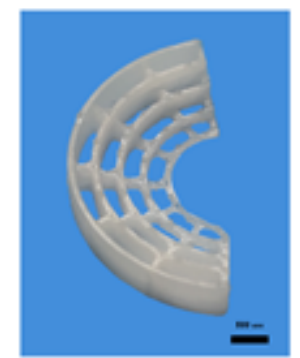

D

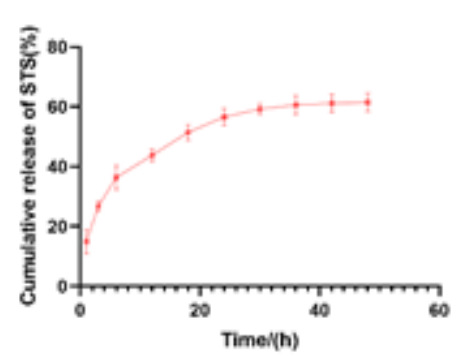


A

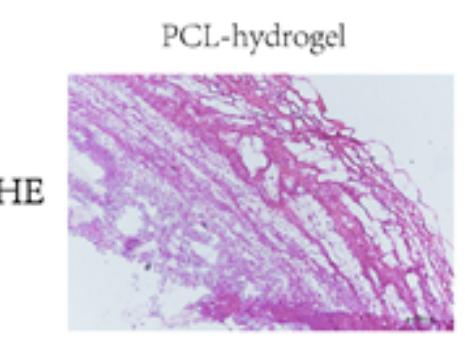

IL-1 $\beta$

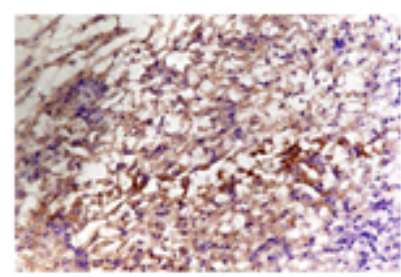

B

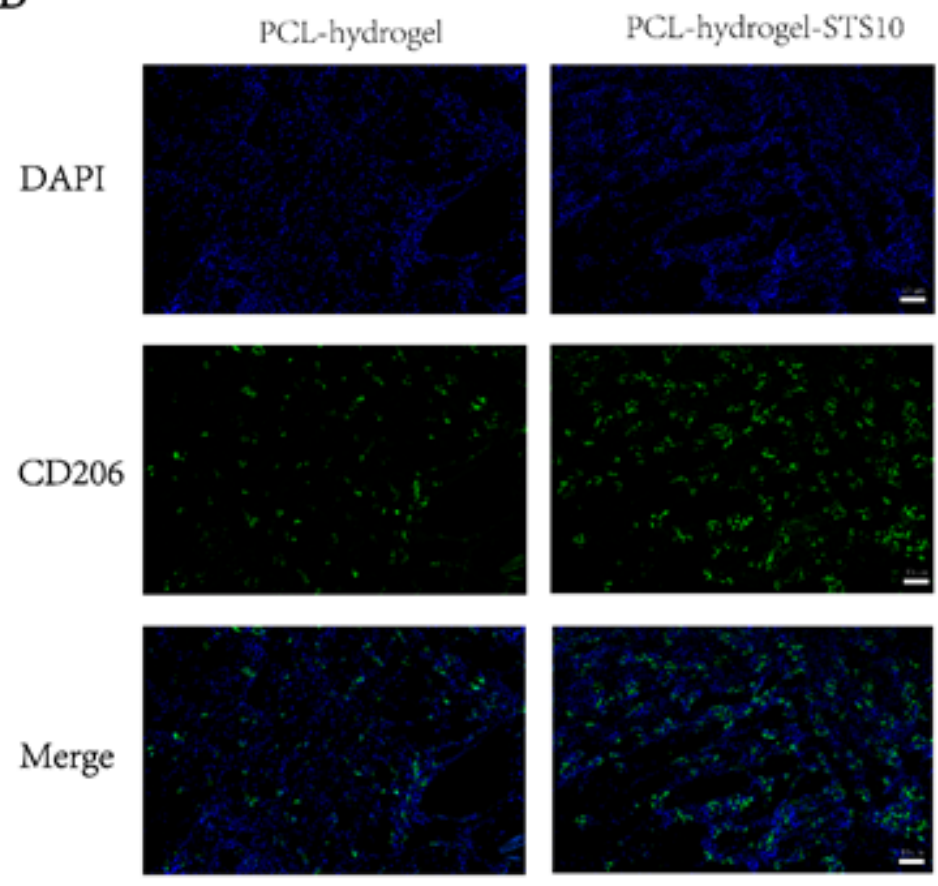

PCL-hydrogel-STS10
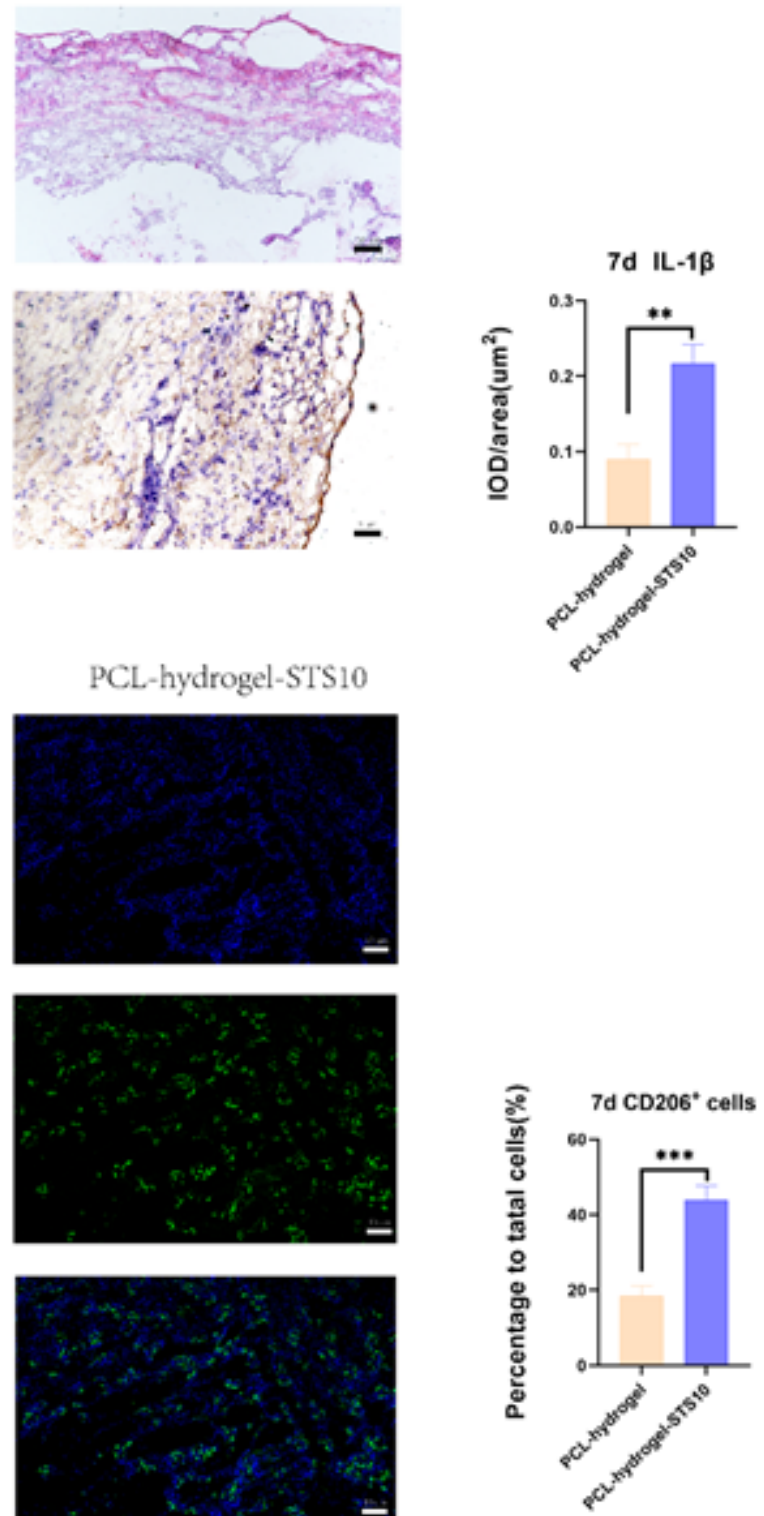

\section{Figure 7}

In vivo assessment of the early host response and macrophage polarization of hybrid scaffolds. (A) H\&E and immunohistochemical staining (IL-1 $\beta$ ) of the hybrid scaffold's immune response in vivo at 7 days.

(B) DAPI and CD206 immunofluorescence staining of the hybrid scaffold's immune response in vivo at 7 days. 
A

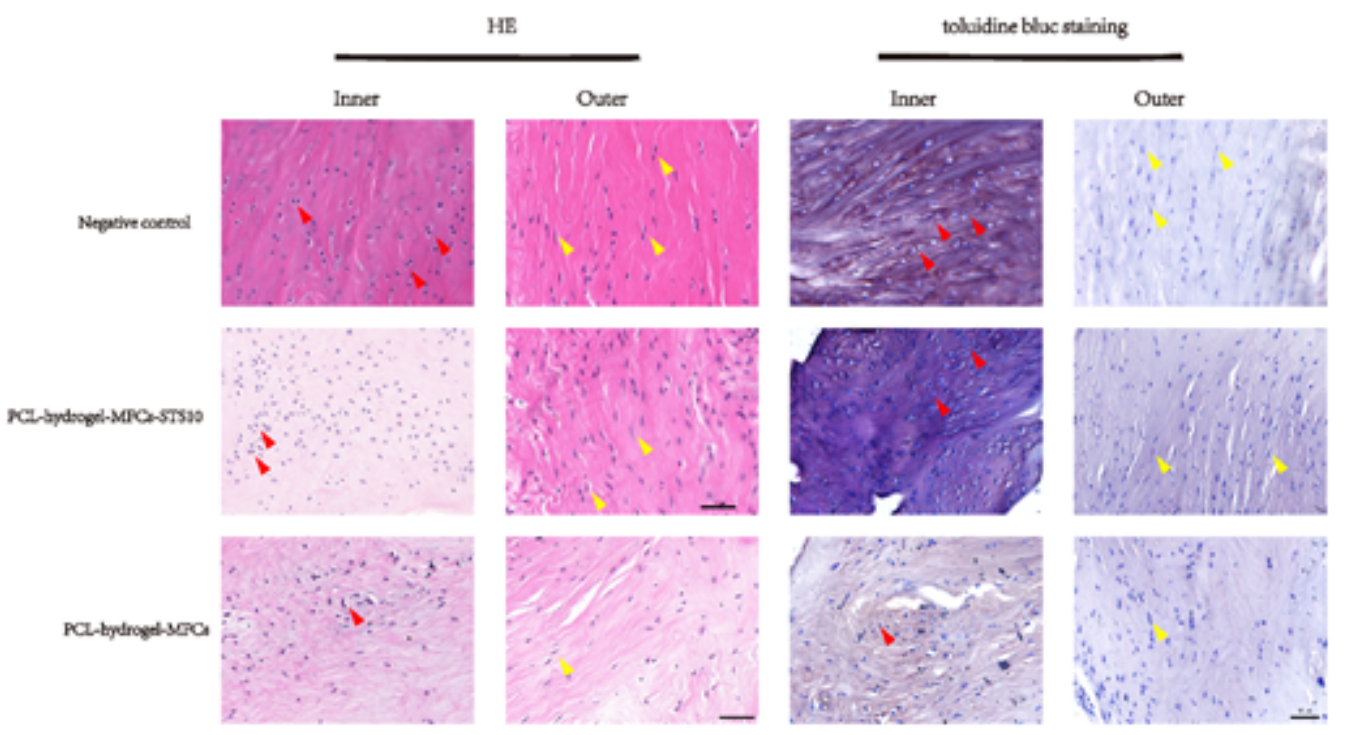

B

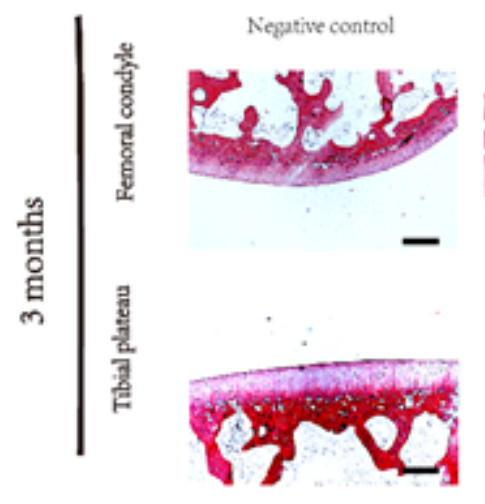

PCL-hydrogel-MFCs-STS10

PCL-hydrogel-MFCs

Positive control
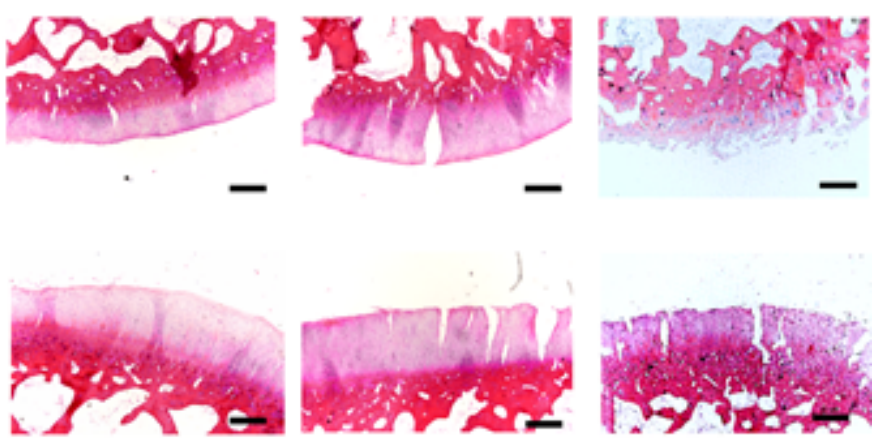

C

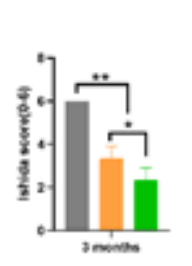

D

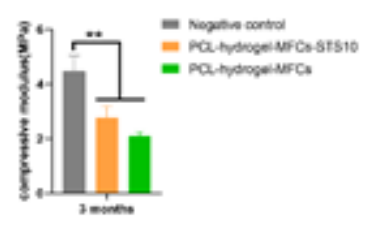

$\mathrm{E}$

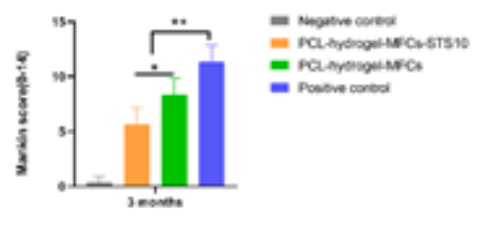

\section{Figure 8}

In vivo repair. (A) H\&E and toluidine blue staining analyses of the regenerated menisci. (B, E) H\&E staining was used to assess the femoral condyle and tibial plateau. (C) Ishida histological score for the regenerated menisci. (D) the compressive modulus of the regenerated menisci. 


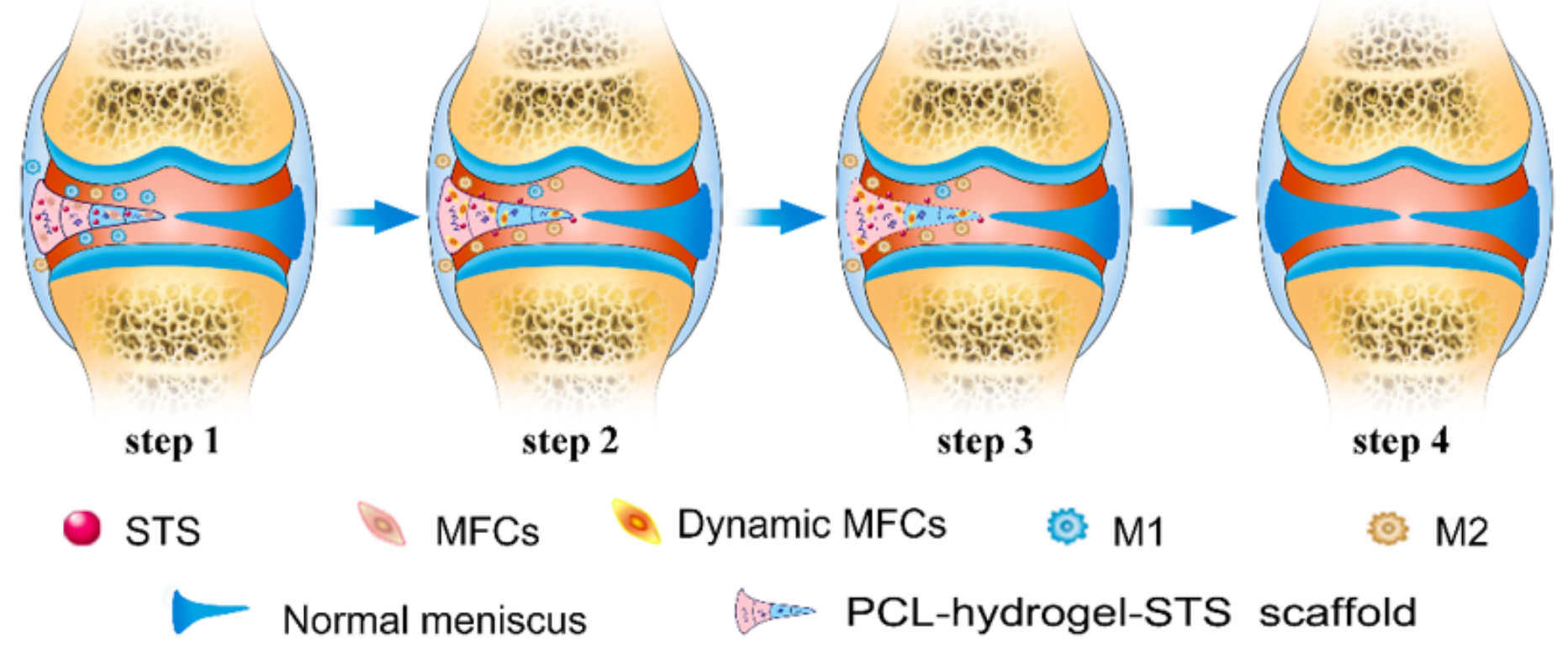

Figure 9

Summarized schematic of regenerative process of the neo-meniscus occurring within STS delivery PCLMECM based hydrogel hybrid scaffold.

\section{Supplementary Files}

This is a list of supplementary files associated with this preprint. Click to download.

- SupplementaryMaterial.docx 